\title{
Uso de los registros de actividad para mejorar la colaboración en línea
}

\section{Use of activity logs to improve online collaboration}

\author{
César Coll Salvador \\ Anna Engel Rocamora \\ Universidad de Barcelona, UB (España) \\ Shamaly Niño Carrasco \\ Universidad Autónoma de Baja California, UABC (México)
}

\section{Resumen}

Se presenta una revisión de trabajos que centran su interés en la explotación de los datos que registran las plataformas de eLearning sobre la actividad de los participantes. Son propuestas que, mediante el análisis de estos datos, generan información que es ofrecida a los estudiantes, en tiempo real, para ayudarles a colaborar y aprender conjuntamente en la plataforma. En esta línea de investigación convergen trabajos de diferentes ámbitos de estudio como las analíticas del aprendizaje, la minería de datos, las herramientas de conciencia de grupo o las herramientas de análisis de la interacción. La revisión adopta una doble perspectiva: i) identificar y analizar los indicadores extraídos de los registros de actividad, su procesamiento, la información generada y las formas de comunicarla; y ii) explorar el modelo y los instrumentos utilizados para valorar el impacto que la información proporcionada tiene sobre los procesos colaborativos en línea y/o los resultados de aprendizaje. Las conclusiones subrayan que, en general, no se explicitan los modelos de aprendizaje colaborativo que fundamentan la selección de los indicadores ni la información comunicada a los estudiantes. También se detectan sesgos importantes como consecuencia de no tener en cuenta la naturaleza multidimensional de los procesos de aprendizaje colaborativo. Además, son pocos los trabajos que analizan las relaciones entre los usos que los estudiantes hacen de la información que reciben y la calidad de los procesos y los resultados del aprendizaje colaborativo, y cuando lo hacen no profundizan en los cambios que la información provoca en las dinámicas grupales.

Palabras clave: analíticas de actividad; aprendizaje en línea; CSCL; enseñanza superior; registros de actividad.

\begin{abstract}
This article presents a review of works that center their interest in eLearning platforms and the data mining of participants' activity. The studies in this research area generate information, through the analysis of such logs and data, that is provided to the students in real time to help them to collaborate and learn through collaboration on the platform. There
\end{abstract}


are studies from different areas of study such as Learning Analytics, Educational Data Mining, Group Awareness Tools or Interaction Analysis Tools. The review takes a double perspective: i) to analyze the data extracted from activity logs, their processing, the information generated and the ways to communicate it; and ii) to explorer the model and the instruments used to assess how the information provided impact on online collaborative processes and/or the learning. The conclusions emphasize that the models of collaborative learning that justifies the selection of the data extracted from the activity logs, the processing, the information generated and provided to the students and the way of communicating it, are not explicitly stated. In addition, important biases are detected because of not considering the multidimensional nature of the collaborative learning processes. Also, few studies analyze the relations between students' uses of the information provided and the quality of their collaborative processes and learning results. The very few studies that do analyze such relation do not go into depth on the changes in group dynamics caused by information.

Keywords: activity analytics; online learning; CSCL; higher education; activity logs.

El uso de las tecnologías digitales para apoyar y guiar los procesos colaborativos de los estudiantes es en la actualidad uno de los focos que más interés despierta en la investigación psicoeducativa (Dillenbourg, Järvelä y Fischer, 2009; Jeong y HmeloSilver, 2016; Stahl, 2015). En este marco, el interés por el "aprendizaje colaborativo mediado por ordenador” (CSCL, por sus siglas en inglés) sigue vigente. Por un lado, estos entornos ofrecen posibilidades inéditas para establecer y mantener la comunicación multidireccional de los estudiantes sin necesidad de que coincidan espacial o temporalmente; por otro lado, las instituciones educativas otorgan una importancia creciente a la capacidad de los estudiantes para trabajar y aprender colaborativamente (Roschelle, 2013).

De acuerdo con Stahl, Koschmann y Suthers (2006), la investigación en el ámbito del CSCL se centra en explorar cómo los entornos mediados por ordenador pueden mejorar la colaboración entre estudiantes y los resultados de su aprendizaje. Sin embargo, distintas revisiones realizadas sobre un conjunto de estudios de CSCL (Dillenbourg et al., 2009; Engel, 2008; Janssen y Bodemer, 2013; Jeong y Hmelo-Silver, 2016; Kirschner y Erkens, 2013; Resta y Laferrière, 2007) evidencian que no toda situación de trabajo colaborativo mediado por ordenador se traduce automáticamenteen dichas mejoras. En efecto, estostrabajos constatanlas dificultades que tienen a menudo los estudiantes para i) participar equitativa y sostenidamente durante todo el proceso colaborativo, ii) compartir una representación común sobre las tareas que tienen que realizar, iii) supervisar el progreso de esas tareas, y iv) involucrarse en procesos de construcción de sistemas de significados cada vez más ricos y compartidos sobre los contenidos de aprendizaje.

De ahí que autores como Janssen y Bodemer (2013) y Jeong y Hmelo-Silver (2016) insistan en aprovechar las características y potencialidades propias y específicas de los entornos CSCL para ayudar a los estudiantes a implicarse en 
procesos colaborativos más eficaces. Ante esta llamada de atención, la preocupación general de una parte importante de los estudios actuales en CSCL se centra en el diseño de entornos digitales que permitan aumentar la probabilidad de que ocurran procesos de construcción conjunta de significados entre los estudiantes (Engel, 2008; Kirschner y Erkens, 2013).

En concreto, encontramos distintas propuestas que coinciden en el interés por desarrollar métodos para explotar el enorme caudal de datos sobre la actividad de los usuarios con el fin de facilitar que sean ellos mismos quienes guíen, orienten y supervisen sus actuaciones para implicarse en procesos colaborativos más eficaces. Es el caso, por ejemplo, de los trabajos que presentan herramientas para desarrollar la conciencia de grupo (Bodemer y Dehler, 2011; Buder, 2011; Janssen y Bodemer, 2013) o que promueven la regulación socialmente compartida (Järvelä et al., 2015). En los apartados siguientes, presentaremos, primero, las características generales y los puntos en común de las distintas propuestas para proceder, seguidamente, a revisar el estado actual de la investigación desarrollada sobre esta cuestión.

\section{LA INFORMACIÓN SOBRE LA ACTIVIDAD DE LOS ESTUDIANTES Y EL USO DE HERRAMIENTAS ANALÍTICAS: ELEMENTOS COMUNES}

De acuerdo con Jeong, Hmelo-Silver y Yu (2014), la interacción que los estudiantes desarrollan en los entornos de CSCL genera una gran cantidad de datos que pueden proporcionar una visión más o menos detallada y situada de los procesos de aprendizaje colaborativo. Desde nuestra consideración, destacan cuatro líneas de investigación que convergen en esa idea:

- la minería de datos educativos -educational data mining (EDM)- (García, Romero, Ventura y de Castro, 2011; Romero y Ventura, 2010), definida como "an emerging discipline, concerned with developing methods for exploring the unique types of data that come from educational settings, and using those methods to better understand students, and the settings in which they learn" http://www.educationaldatamining.org;

- las analíticas del aprendizaje-learning analytics (LA)- (Brown, 2011; Ferguson, 2012; http://www.solaresearch.org), definidas como "the measurement, collection, analysis and reporting of data about learners and their contexts, for purposes of understanding and optimising learning and the environments in which it occurs" (Siemens y Long, 2011, p. 34);

- las herramientas del análisis de la interacción -interaction analysis tools (IA)-, definidas como "the automatic or semi-automatic processes that [...] can serve in order to support the human or artificial actors to take part in the control of the activity, contributing to awareness, self-assessment or even regulation and selfregulation" (Dimitracopoulou, 2008, p. 2); y 
- las herramientas de conciencia de grupo -group awareness tools (GA)(Bodemer y Dehler, 2011; Buder, 2011; Janssen y Bodemer, 2013), definidas como "tools [that] have been developed in order to support users in accessing and utilizing information about their communication partners [...] that even surpasses the face-to-face level” (Bodemer, 2011, p. 1080).

Aunque estas líneas de investigación no comparten necesariamente el CSCL como paradigma de investigación, tienen en común que desarrollan métodos dirigidos a explotar los datos sobre la actividad de los usuarios que registran automáticamente las plataformas educativas, con el fin de comprender mejor y optimizar los procesos de enseñanza y aprendizaje. En todos los casos se trata de recoger datos de las acciones e interacciones de los participantes en el entorno digital, procesarlos, analizarlos en función de ciertas variables y presentar los resultados de ese análisis a los profesores y estudiantes en tiempo real. La finalidad última es que la información brindada guíe y oriente las actuaciones de los estudiantes ayudándoles, por ejemplo, a supervisar y regular su propia actividad o a coordinarse con el resto de miembros del grupo durante la realización de las actividades de aprendizaje; o bien que los docentes la utilicen para orientar sus intervenciones, para tomar decisiones sobre cuándo sus estudiantes necesitan apoyos específicos o para detectar incomprensiones o patrones de actuación irregulares.

Inicialmente, estas líneas de investigación se centraron en superar las limitaciones de la comunicación en línea tratando de proporcionar informaciones similares a las que proporciona la comunicación cara a cara (Janssen y Bodemer, 2013), a partir de datos básicos como el número de sesiones iniciadas, las contribuciones en los foros o el tiempo dedicado a consultar los recursos de la plataforma (Dawson, Gaševi, Siemens y Joksimovic, 2014).

Las propuestas más recientes van dirigidas a proporcionar informaciones que no son directamente observables y que pueden hacer la comunicación y la colaboración en línea más ricas de lo que pueden serlo en situaciones cara a cara. Así, se han diseñado sistemas que, por ejemplo, proporcionan información acerca de cómo el grupo evalúa las contribuciones de sus miembros o sobre los conocimientos previos que los participantes tienen sobre los contenidos de enseñanza (Bodemer y Dehler, 2011; Buder, 2011).

Sin embargo, pese a tener metas e intereses comunes, las distintas líneas de investigación mencionadas difieren en algunos aspectos importantes. Más que las diferencias tecnológicas y técnicas del diseño de estas herramientas, lo que nos interesa destacar aquí son las diferencias derivadas de los enfoques sobre la enseñanza, el aprendizaje y la colaboración en los que se apoyan, ya que consideramos que estos enfoques condicionan en gran medida los aspectos de los procesos y los resultados del CSCL que se pretenden mejorar con el uso de las herramientas y, con ello, el tipo de información que se recoge del entorno y que se ofrece, en concreto, a los estudiantes. 
Para dar cuenta de lo anterior, hemos realizado una revisión bibliográfica de la literatura centrada en las cuatro líneas de investigación descritas. El procedimiento y los resultados de esta revisión se presentan en los siguientes apartados.

\section{METODOLOGÍA}

La revisión de la literatura se realizó en dos fases, según el procedimiento sugerido por Cooper (1988). En primer lugar, realizamos una búsqueda en la base de datos Scopus utilizando la siguiente combinación de términos: "learning analytics" OR "big data" OR "group awareness" OR "interaction analysis" OR "educational data mining" AND "higher education" OR "online learning" OR "blended learning" OR "e-learning" OR "computer-supported collaborative learning" AND DOCTYPE "article", sin limitar la búsqueda a un periodo específico. La búsqueda realizada en septiembre de 2017 arrojó 358 resultados (véase la tabla 1).

Tabla 1. Resultados de la búsqueda realizada agrupados por año de publicación

\begin{tabular}{|l|c|}
\hline \multicolumn{1}{|c|}{ Año de publicación } & Núm. de artículos \\
\hline 2017 & 10 \\
\hline 2016 & 109 \\
\hline 2015 & 95 \\
\hline 2014 & 46 \\
\hline 2013 & 25 \\
\hline $2012-2008$ & 61 \\
\hline anteriores a 2007 & 12 \\
\hline Total & 358 \\
\hline
\end{tabular}

En segundo lugar, procedimos al escrutinio del resumen y palabras clave de los 358 documentos siguiendo los siguientes criterios de inclusión/exclusión:

- trabajos que remiten a experiencias desarrolladas o diseñadas para educación superior;

- trabajos en los que la información proporcionada por las herramientas va dirigida a los estudiantes;

- trabajos que describen el diseño, análisis y/o implementación de la herramienta;

- trabajos en los que el uso de la herramienta implica la resolución de tareas colaborativas en línea;

- trabajos que no son revisiones de otros trabajos. 
La aplicación de estos criterios nos ha llevado a descartar 341 trabajos (ver tabla 2), únicamente 17 trabajos (4,75\%) cumplieron con los criterios establecidos.

Tabla 2. Número de trabajos descartados de la revisión de la literatura

\begin{tabular}{|l|c|}
\hline \multicolumn{1}{|c|}{ Categoría } & N \\
\hline Presentan herramientas no diseñadas para educación superior & 13 \\
\hline Presentan herramientas que entregan información a profesores & 11 \\
\hline Presentan herramientas que entregan información a nivel institucional & 105 \\
\hline Presentan herramientas para la toma de decisiones territorial o nacional & 21 \\
\hline Presentan herramientas no diseñadas para el ámbito educativo & 164 \\
\hline Revisiones de otros trabajos & 27 \\
\hline Total & 341 \\
\hline
\end{tabular}

A continuación, presentamos los resultados de la revisión de estos 17 trabajos atendiendo a cuatro aspectos: (i) dimensiones de la actividad colaborativa sobre las que se informa a los estudiantes y modelo de aprendizaje colaborativo que subyace a la selección de dichas informaciones; (ii) cómo se recoge la información y cómo se presenta a los estudiantes, (iii) cuándo o en qué momento se informa a los estudiantes; y (iv) cuál es el modelo y/o los instrumentos utilizados para valorar el impacto de la información proporcionada sobre los procesos colaborativos y los resultados de aprendizaje.

\section{RESULTADOS}

\section{Dimensiones sobre las que se informa y marco teórico subyacente}

La información que se entrega a los participantes puede versar sobre cinco dimensiones de la actividad colaborativa: i) el proceso de construcción de significados que los estudiantes desarrollan sobre los contenidos de enseñanza y aprendizaje; ii) su nivel de participación; iii) los elementos relacionados con el abordaje y desarrollo de la tarea; iv) los aspectos socio-afectivos y las dinámicas comunicativas o motivacionales de los estudiantes; y v) los elementos metacognitivos de regulación que los estudiantes adoptan para desarrollar la tarea.

Desde la primera dimensión -construcción de significados-, encontramos herramientas interesadas por el contenido de las contribuciones de los participantes. Así, la herramienta que propone Bodemer (2011) permite a los estudiantes que trabajan en díadas conocer el estado o nivel de avance del desarrollo de la tarea de la pareja, mediante la visualización compartida de las pantallas de los ordenadores. De modo que, ayudados también por una herramienta de chat, los estudiantes tienen 
la posibilidad de discutir y corregir los errores identificados en el manejo de los contenidos de la pareja. Otro ejemplo de este tipo de herramientas lo encontramos en el trabajo de Marzouk et al. (2016). En este caso, el estudiante recibe información sobre los conceptos que él mismo va creando y señalando como conceptos clave en las diferentes actividades que desarrolla, con el fin de que vincule los contenidos de los distintos temas de la asignatura.

Desde la dimensión de participación, se proporciona información sobre el nivel de implicación de los estudiantes, es decir, sobre qué están haciendo en cada momento y su contribución a la realización de la tarea conjunta. Típicamente son herramientas que ofrecen información cuantitativa como el número de accesos a la plataforma, los recursos utilizados y la frecuencia de uso (Bravo, Redondo, Verdejo y Ortega, 2008; Park y Jo, 2015; Pifarré, Cobos y Argelagós, 2014; Zorrilla, Álvarez y GarcíaSaiz, 2015), el número de contribuciones (Fessakis, Dimitracopoulou y Palaiodimos, 2013; Wise, Vytasek, Hausknecht y Zhao, 2016; Xia, 2015), o las entradas aportadas por cada participante a una base de datos compartida (Kimmerle y Cress, 2008).

Desde la tercera dimensión, abordaje y desarrollo de la tarea, encontramos herramientas que ofrecen información relacionada con los conocimientos previos que poseen los estudiantes sobre el contenido específico que trabajarán conjuntamente. Así, la Knowledge awareness tool (KAT) (Sangin, Molinari, Nüssli y Dillenbourg, 2011) informa sobre el nivel de comprensión que los estudiantes tienen cuando elaboran, organizados en díadas, un mapa conceptual, de tal manera que conozcan el nivel de dominio que tienen sobre un tema y puedan, de este modo, desarrollar estrategias para un mejor abordaje y desarrollo de la tarea.

Desde la dimensión socioafectiva, encontramos herramientas que informan sobre aspectos relacionados con la motivación de los participantes o las dinámicas comunicativas en las que se implican. Por ejemplo, Schoor, Kownatzki, Narciss y Körndle (2014) proponen una herramienta que ofrece a los estudiantes un gráfico de líneas que muestra su índice de motivación individual en cada momento. También es muy frecuente la utilización de técnicas de análisis de redes sociales para describir los patrones de interacción y las dinámicas comunicativas que establecen los participantes (Kuosa et al., 2016; McCormick, 2013).

Con respecto a la última dimensión -elementos metacognitivos de regulación-, Kwon, Hong y Laffey (2013) proponen una herramienta (Metacognitive Teamwork Management Tool-MTMT) que ofrece información relativa a los tres procesos principales de regulación: planificación, seguimiento y evaluación. En cada fase, la MTMT permite a los estudiantes evaluar su desempeño grupal y compartir la información con los otros miembros del grupo.

Excepcionalmente, en el trabajo de Mora, Caballé y Daradoumis (2016) encontramos un buen ejemplo de herramienta que informa sobre los aspectos relacionados con dos dimensiones: la construcción de significados y la socioafectiva, ya que ofrece información sobre la naturaleza y la calidad de las interacciones 
colaborativas (pasivas, proactivas, reactivas), así como sobre el número de relaciones que establecen los estudiantes mediante sus intercambios comunicativos.

$\mathrm{Al}$ analizar los aspectos centrales del proceso de colaboración que las distintas herramientas destacan en el marco teórico y que subyacen a la elección del tipo de información que entregan, es posible identificar hasta cuatro tipos de trabajos distintos: (i) el modelo teórico que subyace es un modelo de aprendizaje pero no de aprendizaje colaborativo; (ii) el modelo que subyace remite al aprendizaje colaborativo pero se centra solo en una dimensión; (iii) el modelo remite a un modelo de aprendizaje colaborativo completo pero los indicadores se centran en una o dos dimensiones; y (iv) no se explicita ningún elemento o aspecto teórico del aprendizaje en general o del aprendizaje colaborativo en particular.

Dos ejemplos del primer tipo de trabajos son las publicaciones de McCormick (2013) y Mora et al. (2016), que mencionan, sin detallar, algunos componentes teóricos de una visión general del aprendizaje a partir de la cual proceden a seleccionar los aspectos de la actividad sobre los que informan a los estudiantes. Con respecto a los trabajos que cuentan con un marco teórico concreto sobre el aprendizaje colaborativo, dos ejemplos representativos son las propuestas de Fessakis et al. (2013) y Schoor et al. (2014), si bien el abordaje empírico que realizan se centra en una sola dimensión. Mientras que en el primero de los trabajos, el foco se centra en la participación de los estudiantes, en el segundo se destaca la importancia de la motivación grupal para el desarrollo de los procesos colaborativos.

En el tercer tipo de trabajos, encontramos seis publicaciones (Bodemer, 2011; Kimmerle y Cress, 2008; Kwon et al., 2013; Marzouk et al., 2010; Pifarré et al., 2014; Sangin et al., 2011) que parten de un modelo de aprendizaje colaborativo completo que contempla de dos a tres dimensiones de la actividad colaborativa, pero las informaciones que finalmente entregan a los estudiantes versan solo sobre una o dos dimensiones. Por mencionar solo un ejemplo, Kwon et al. (2013) reconocen que la interdependencia positiva entre los miembros del grupo es determinante para que coordinen sus esfuerzos para completar la tarea. Sin embargo, para que esta coordinación efectivamente sea conjunta, es necesario desarrollar la conciencia grupal sobre lo que todos los miembros del grupo hacen, saben y entienden, favoreciendo con ello el desarrollo de estrategias de regulación grupal para mejorar el proceso colaborativo y los resultados de aprendizaje. Cuando se revisa en detalle la propuesta de herramienta, se observa que la información entregada a los estudiantes se basa en las valoraciones que los propios estudiantes hacen sobre el desempeño grupal.

Finalmente, la gran mayoría de las publicaciones revisadas corresponden a la cuarta categoría (Bravo et al., 2008; Kuosa et al., 2016; Park y Jo, 2015; Silius, Tervakari y Kailanto, 2013; Wise et al., 2016; Xia, 2015; Zorrilla et al., 2015), que se distingue por la falta de un marco teórico claro sobre el aprendizaje en general o sobre el aprendizaje colaborativo en particular. 


\section{Recolección de datos y formatos de visualización}

Acerca de los datos de la actividad de los participantes que se recogen, encontramos propuestas que obtienen la información de manera automática del entorno digital y otros que la solicitan directamente a los estudiantes. En el primer caso, los registros de actividad son procesados para proporcionar información a los estudiantes sobre su participación y nivel de implicación; la mayoría de los trabajos revisados proceden de esta manera (Bravo et al., 2008; Fessakis et al., 2013; Kimmerle y Cress, 2008; Kuosa et al., 2016; Marzouk et al., 2016; McCormick, 2013; Mora et al., 2016; Park y Jo, 2015; Pifarré et al., 2014; Silius et al., 2013; Wise et al., 2016; Xia, 2015; Zorrilla et al., 2015). En el segundo, utilizado en pocos trabajos, la información se obtiene directamente de los estudiantes, ya sea administrando un pre-test para evaluar los conocimientos previos del grupo (Sangin et al., 2011), o bien cuestionarios para valorar su motivación o implicación en la tarea académica (Kwon et al., 2013; Schoor et al., 2014).

En relación con la forma de entrega, la gran mayoría de los trabajos opta por proporcionar la información a los estudiantes individuales. Algunos les proporcionan información tanto de su propia actividad como de la actividad de otro(s) estudiante(s), ya sea que trabajen en díadas o tríos (Bodemer, 2011; Sangin et al., 2011; Schoor et al., 2014) o en grupos más amplios (Fessakis et al., 2013; Kimmerle y Cress, 2008; Kuosa et al., 2016; Kwon et al., 2013; Mora et al., 2016; Park y Jo, 2015; Zorrilla et al., 2015). Otros trabajos, en cambio, ofrecen a cada estudiante solo la información de su propia actividad (Bravo et al., 2008; Marzouk et al., 2016). Únicamente hemos identificado una herramienta que ofrece al grupo la información derivada del análisis de la actividad colectiva y no proporciona ningún tipo de información de forma individual (Kwon et al., 2013).

Las herramientas difieren también en la entrega de la información en función de, al menos, dos aspectos. El primero se relaciona con los formatos de visualización que utilizan; y el segundo con la entrega de orientaciones relativas a cómo debe interpretarse la información. Los formatos de visualización empleados son muy diversos y generalmente son sencillos y de fácil interpretación. Así, algunas herramientas optan por utilizar gráficos simples de líneas (Schoor et al., 2014), de barras (Sangin et al., 2011), de columnas o histogramas (Bravo et al., 2008; Kimmerle y Cress, 2008; Kuosa et al., 2016), esferas cuyo tamaño está relacionado con el número de contribuciones (Fessakis et al., 2013), dispersogramas (Park y Jo, 2015), gráficas radiales (Zorrilla et al., 2015) o incluso sociogramas representando la posición de los participantes en una red de relaciones (McCormick, 2013). Otras herramientas presentan visualizaciones más interactivas, como la propuesta por Bodemer (2011) en la que las díadas de estudiantes pueden ver representada, en tiempo real, la actividad colaborativa que desarrollan mediante la visualización compartida de las pantallas de los ordenadores. Excepcionalmente, algunas herramientas ofrecen orientaciones para interpretar las informaciones ofrecidas como, por ejemplo, la 
herramienta propuesta por Mora et al. (2016) en la que los indicadores de actividad se iluminan de diferentes colores en función de su lejanía o proximidad a un perfil ideal de estudiante (ver figura 1).

Figura 1. Ejemplo de una herramienta que ofrece los indicadores de actividad coloreados en función de un determinado perfil de actividad

SLO cOn SNA

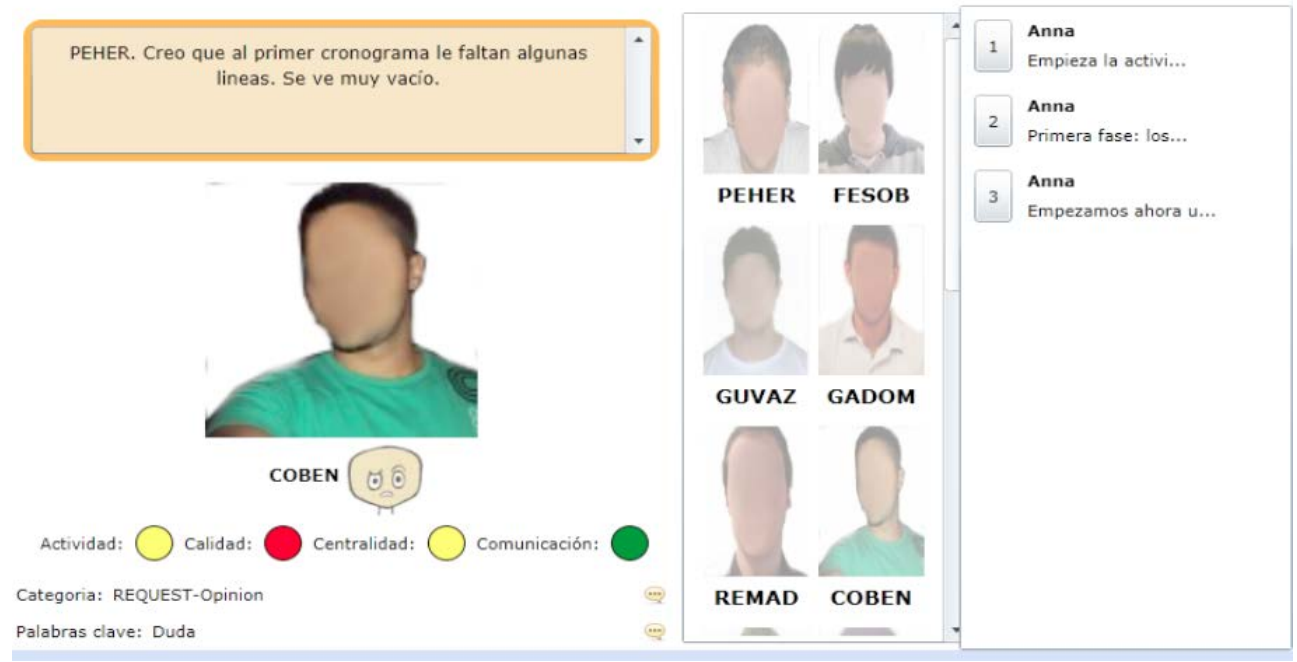

Fuente: Mora et al., 2016, p. 46.

\section{Momento de entrega de la información}

Respecto a cuándo o en qué momento se entrega la información, encontramos herramientas que permiten que la información sea permanentemente visible y otras que la ofrecen de manera periódica o a demanda de los estudiantes. Muchas de las herramientas que entregan la información en tiempo real no ofrecen a los usuarios la posibilidad de ocultarla. Así, las propuestas de Bodemer (2011), Sangin et al. (2011) y Schoor et al. (2014) obligan a que la información esté permanentemente visible. En otras herramientas, la información aparece bajo demanda de los estudiantes (Bravo et al., 2008; Kuosa et al., 2016; Park y Jo, 2015; Pifarré et al., 2014; Wise et al., 2016), quienes pueden solicitarla de manera individual en cualquier momento. En pocas herramientas, la información aparece en un momento predeterminado, como en la herramienta propuesta por Kwon et al. (2013), que ofrece la información a los estudiantes cada vez que termina una fase del proceso colaborativo (ver figura 2), 
permitiéndoles conocer las valoraciones que el resto de sus compañeros hacen sobre la actividad de la fase que acaba de concluir.

Figura 2. Ejemplo de una herramienta que ofrece la información a los estudiantes al término de cada fase

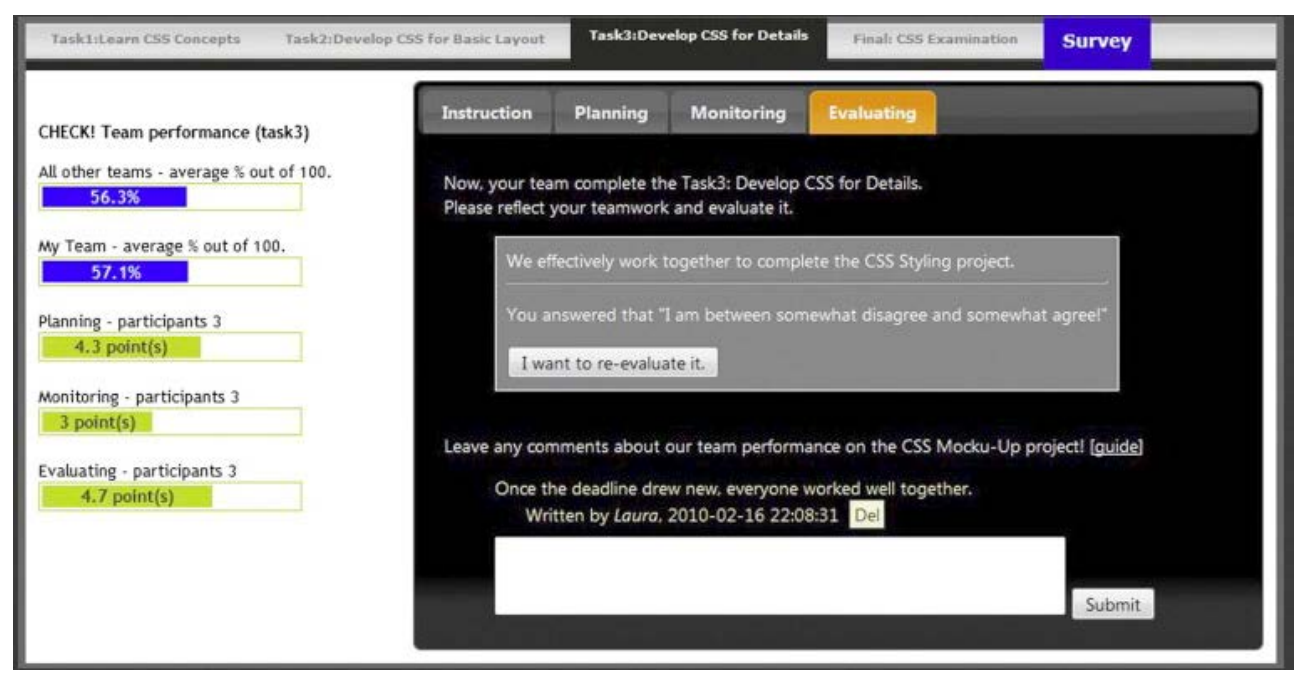

Fuente: Kwon et al., 2013, p. 1276.

\section{Instrumentos utilizados para valorar el impacto de la información}

El análisis de los procedimientos de análisis utilizados para valorar el impacto de la información proporcionada nos ha permitido identificar hasta cuatro tipos de trabajos (ver tabla 3).

Tabla 3. Métodos e instrumentos utilizados para valorar el impacto de las herramientas

\begin{tabular}{|l|l|l|}
\hline $\begin{array}{l}\text { Método o instrumento utilizado } \\
\text { para valorar el impacto }\end{array}$ & $\begin{array}{c}\text { Aspecto sobre el que se analiza } \\
\text { el impacto }\end{array}$ & \multicolumn{1}{c|}{ Referencia } \\
\hline $\begin{array}{l}\text { pre-test y post-test } \\
\text { grupos control y experimental }\end{array}$ & $\begin{array}{l}\text { mejora de los resultados de } \\
\text { aprendizaje }\end{array}$ & $\begin{array}{l}\text { Bodemer, 2011 } \\
\text { Kwon et al., 2013 } \\
\text { Mora et al., 2016 } \\
\text { Sangin et al., 2011 } \\
\text { Schoor et al., 2014 }\end{array}$ \\
\hline $\begin{array}{l}\text { valores de los indicadores } \\
\text { grupos control y experimental }\end{array}$ & $\begin{array}{l}\text { mejora del proceso de } \\
\text { colaboración }\end{array}$ & Fessakis et al., 2013 \\
\hline
\end{tabular}




\begin{tabular}{|l|l|l|}
\hline $\begin{array}{l}\text { Método o instrumento utilizado } \\
\text { para valorar el impacto }\end{array}$ & $\begin{array}{c}\text { Aspecto sobre el que se analiza } \\
\text { el impacto }\end{array}$ & \multicolumn{1}{|c|}{ Referencia } \\
\hline $\begin{array}{l}\text { calidad de los productos } \\
\text { grupales y calificaciones finales } \\
\text { individuales } \\
\text { grupos control y experimental }\end{array}$ & $\begin{array}{l}\text { mejora de los resultados de } \\
\text { aprendizaje y del proceso de } \\
\text { colaboración }\end{array}$ & Pifarré et al., 2014 \\
\hline $\begin{array}{l}\text { cuestionarios o encuestas de } \\
\text { valoración }\end{array}$ & $\begin{array}{l}\text { utilidad de las herramientas o } \\
\text { satisfacción de los estudiantes }\end{array}$ & $\begin{array}{l}\text { Bravo et al., 2008 } \\
\text { Kimmerle y Cress, 2008 } \\
\text { Kuosa et al., 2016 } \\
\text { Marzouk et al., 2016 } \\
\text { McCormick, 2013 } \\
\text { Park y Jo, 2015 } \\
\text { Silius et al., 2013 } \\
\text { Wise et al., 2016 } \\
\text { Xia, 2015 } \\
\text { Zorrilla et al., 2015 }\end{array}$ \\
\hline
\end{tabular}

En primer lugar, encontramos un conjunto de trabajos caracterizados por administrar pre-tests y post-tests para valorar los conocimientos adquiridos por los estudiantes; de esta manera, a partir de las diferencias estadísticamente significativas entre los valores obtenidos por el grupo control y el grupo experimental se relaciona el impacto de la información entregada con la mejora de los resultados de aprendizaje.

Encontramos un segundo tipo de estudios que toman los propios valores de los indicadores que se entregaron a los estudiantes como instrumentos para analizar la efectividad de la herramienta. De entre los trabajos revisados, solo hallamos uno que presenta esta característica (Fessakis et al., 2013). Al igual que el primer tipo de estudios, también utiliza métodos estadísticos para establecer diferencias significativas, en este caso entre el número de contribuciones que realiza el grupo control y el grupo experimental, si bien estas diferencias se asocian con el impacto de la herramienta sobre los procesos colaborativos.

Los estudios del tercer tipo (Pifarré et al., 2014) se caracterizan por el uso de dos instrumentos de análisis de diferente naturaleza: por un lado, consideran la codificación de los productos grupales generados para valorar su calidad según la diversidad de dimensiones de aprendizaje que presentan y, por otro lado, consideran las calificaciones finales obtenidas por los estudiantes; en ambos casos, se contrastan los valores obtenidos por el grupo control y el grupo experimental. Es importante señalar que, entre todos los trabajos de la revisión, este es el único que analiza, al mismo tiempo, el impacto que la herramienta tiene sobre los procesos colaborativos y los resultados de aprendizaje.

Finalmente, los trabajos incluidos en el cuarto grupo se distinguen de los anteriores porque centran su interés en recoger y analizar las valoraciones sobre la utilidad de las herramientas diseñadas y la satisfacción de los estudiantes cuando las usan. 


\section{DISCUSIÓN Y CONCLUSIONES}

La revisión realizada resalta el interés en profundizar en el análisis del impacto que la información proporcionada a los estudiantes sobre algunos aspectos de su actividad, en un entorno CSCL, tiene sobre los procesos colaborativos. En el conjunto de trabajos referenciados, también es posible identificar una serie de desafíos que, a nuestro juicio, deben enfrentarse para seguir avanzando en este ámbito de investigación. Entre estos desafíos destacan los relativos a: i) el diseño de las propias herramientas, ii) el diseño de las situaciones en las que se utiliza la herramienta y iii) los procedimientos de análisis utilizados para valorar su impacto en los procesos y resultados de aprendizaje de los estudiantes.

En relación con el primer desafío, el diseño de las herramientas, hace una década Dimitracopoulou (2008) ya llamaba la atención sobre el hecho de que la mayor parte de las herramientas se basan en indicadores de la actividad que no requieren ningún tipo de procesamiento, como el número de accesos al entorno, el número de contribuciones escritas, el porcentaje de las mismas que reciben respuesta, etc. De los trabajos revisados, muy pocas herramientas cuentan con indicadores de alto poder interpretativo o combinaciones de índices de distinto tipo que ofrezcan una representación más rica y multidimensional de la actividad de cada uno de los participantes, o del grupo en su conjunto, susceptible de ser contrastada con una visión del aprendizaje, de la colaboración o del aprendizaje colaborativo. De hecho, en el conjunto de trabajos revisados, hemos identificado, excepcionalmente, propuestas que remiten a aspectos metacognitivos (Kwon et al., 2013), o que combinan indicadores relacionados con dos dimensiones de la actividad colaborativa (Mora et al., 2016).

En el mismo sentido, coincidimos con Bravo et al. (2008) al señalar que las propuestas se han centrado, principalmente, en presentar información sobre el análisis de las acciones o participación de los alumnos y mucho menos en presentar información sobre el proceso de construcción colaborativa del conocimiento.

En relación con el segundo desafío, las situaciones estudiadas, nuestra revisión pone de manifiesto, igual que otros autores (Buder, 2011; Janssen y Bodemer, 2013), que la gran mayoría de los estudios se llevan a cabo en condiciones artificiales y de muy corta duración, típicamente una única actividad de enseñanza y aprendizaje en una sesión que oscila entre los 40 y 120 minutos y en la que participan díadas o tríos (Bodemer, 2011; Kwon et al., 2013; Sangin et al., 2011; Schoor et al., 2014). En este sentido, coincidimos con Dimitracopoulou (2008) y Kimmerle y Cress (2008) en la necesidad de realizar estudios en situaciones naturales, con una duración temporal más larga, que permita captar el impacto de la información a medida que evolucionan los procesos colaborativos, y en grupos más grandes que los conformados por dos o tres estudiantes, como lo hacen, por ejemplo, Fessakis et al. (2013) y Pifarré et al. (2014). 
El último desafío hace referencia a los procedimientos de análisis utilizados para valorar el impacto de la información proporcionada por estas herramientas en los procesos colaborativos y los resultados de aprendizaje. Hemos encontrado algunos trabajos que asocian el incremento de contribuciones o de las relaciones entre los miembros del grupo (Fessakis et al., 2013) con la mejora de los procesos colaborativos, o bien la mejora de los resultados en los post-tests con los aprendizajes logrados (Bodemer, 2011; Kwon et al., 2013; Sangin et al., 2011), pero en ninguno de los casos encontramos que se profundice en el análisis de los cambios que la información provoca en las dinámicas de participación y actuación conjunta que establecen los estudiantes.

$\mathrm{Al}$ respecto, Buder (2011) señala la urgencia de ir más allá e investigar las complejas interacciones que se producen entre, por una parte, las características de la información que se proporciona, las particularidades del grupo, el tipo de tareas $\mathrm{y}$ contenidos de enseñanza y aprendizaje que se proponen $\mathrm{y}$, por otra, los procesos colaborativos y los resultados de aprendizaje.

Enfrentar los desafíos señalados y abrir nuevas perspectivas en la investigación en este ámbito requiere, en nuestra opinión, superar los enfoques de carácter más tecnológico que psicoeducativo adoptados por buena parte de las investigaciones, en las que se pone de manifiesto la ausencia de un marco teórico claro sobre el aprendizaje en general, o sobre el aprendizaje colaborativo en particular, que fundamente, por una parte, la selección de los aspectos o componentes de la actividad sobre los que se va a informar a los participantes y, por otra, el modelo y los instrumentos de análisis mediante los cuales valorar el impacto que el uso de esta información tiene sobre los procesos de colaboración y sobre el aprendizaje.

En este sentido, coincidimos con diversos autores (Dehler et al., 2011; Dawson et al., 2014; Jeong y Hmelo-Silver, 2016) en la urgencia de diseñar herramientas que puedan contribuir efectivamente a mejorar el aprendizaje colaborativo en línea, proporcionando a los estudiantes una imagen situada y multidimensional de sus procesos colaborativos. Como puntualizan Brown (2012) y Ferguson (2012), tal desarrollo requiere disponer de un modelo teórico potente que ayude a decidir qué información hay que recopilar y procesar, cómo hay que interpretarla y cómo promover, a partir de esa información, actuaciones dirigidas a mejorar los procesos y los resultados del aprendizaje colaborativo.

Desde nuestra consideración, una herramienta diseñada con las características anteriores y para tales fines debe cumplir con, al menos, tres exigencias sustanciales. En primer lugar, debe posibilitar un análisis de los datos de alto nivel que permita definir perfiles diferenciados de la actividad de los estudiantes a partir delos aspectoso dimensiones que teóricamente se consideren esenciales para el desarrollo efectivo de los procesos colaborativos, lo cual implica contar con un perfil de colaboración idóneo con el que los estudiantes puedan contrastar sus perfiles de actividad. En segundo lugar, debe presentar la información en formatos de visualización sencillos y de fácil comprensión para los estudiantes, de tal manera que la información presentada por 
la herramienta sea una ayuda efectiva. Y, en tercer lugar, la herramienta debe ser lo suficientemente flexible para ser utilizada en una gama amplia de entornos de aprendizaje en línea en distintos tipos de actividades y con grupos de estudiantes de distintas características, es decir, debe permitir confeccionar perfiles de actividad adecuados a las características concretas de la situación de enseñanza y aprendizaje.

En esta dirección se orientan los constructos teóricos y metodológicos del modelo de influencia educativa distribuida (Coll, Bustos y Engel, 2011; Coll, Bustos, Engel, de Gispert y Rochera, 2013; Coll, Engel y Bustos, 2009). Los resultados de algunos trabajos empíricos realizados desde esta perspectiva (Coll, Bustos y Engel, 2015; Coll, Engel y Niño, 2017; Niño, 2017) indican que el éxito de la colaboración y sus resultados aparecen asociados al grado en que los participantes en los procesos de aprendizaje colaborativo asumen, en conjunto, su responsabilidad como receptores y dispensadores de ayuda para el resto.

Así mismo, y para concluir, como Ferguson (2012) sugiere, futuras investigaciones en este ámbito deben adoptar un enfoque multidisciplinar que conjugue la visión de la psicología de la educación sobre los procesos de aprendizaje colaborativo y el potencial de cálculo de los sistemas informáticos para generar de forma automática información sobre la actividad de los participantes y proporcionarles acceso en tiempo real a esta información.

\section{REFERENCIAS BIBLIOGRÁFICAS}

Bodemer, D. (2011). Tacit guidance for collaborative multimedia learning. Computers in Human Behavior. 27(3), 1079-1086. doi: https://doi. org/10.1016/j.chb.2010.05.016

Bodemer, D., y Dehler, J. (2011). Group awareness in CSCL environments. Computers in Human Behavior, 27(3), 1043-1045. doi:https://doi.org/10.1016/j. chb.2010.07.014

Bravo, C., Redondo, M. A., Verdejo, M. F., y Ortega, M. (2008). A framework for process-solution analysis in collaborative learning environments. International Journal of Human Computer Studies, 66(11), 812-832. doi: https://doi. org/10.1016/j.ijhcs.2008.08.003

Brown, M. (2011). Learning Analytics: The Coming Third Wave. EDUCAUSE Learning Initiative Brief, 1(4), 1-4. Recuperado de http://www.educause.edu/blog/ pkurkowski/ELIReleasesNewBriefonLearningA/229163
Buder, J. (2011). Group awareness tools for learning: Current and future directions. Computers in Human Behavior, 27(3), 1114-1117. doi: https://doi.org/10.1016/j. chb.2010.07.012

Coll, C., Bustos, A., y Engel, A. (2011). Perfiles de participación y presencia docente distribuida en redes asíncronas de aprendizaje: la articulación del análisis estructural y de contenido. Revista de Educación, 354. 657-688. Recuperado de http://www.mecd.gob.es/dctm/revistade-educacion/articulos-re354/re35426. pdf?documentId=0901e72b811e1d42

Coll, C., Bustos, A., y Engel, A. (2015). La información sobre el ejercicio de la influencia educativa como medio para favorecer la participación y el aprendizaje en un foro en línea. Infancia y Aprendizaje, 38, 368-401. doi: https:// doi.org/10.1080/02103702.2015.1016745 Coll, C., Bustos, A., Engel, A., de Gispert, I., y Rochera, M. J. (2013). Distributed 
Educational Influence and ComputerSupported Collaborative Learning. Digital Education Review, 24, 23-42. Recuperado de http://revistes.ub.edu/ index.php/der/article/view/11274/pdf

Coll, C., Engel, A., y Bustos, A. (2009). Distributed Teaching Presence and Participants' Activity Profiles: a theoretical approach to the structural analysis of Asynchronous Learning Networks. European Journal of Education, 44(4), 521-538. doi: https://doi.org/10.1111 i.1465-3435.2009.01406.X

Coll, C., Engel, A., y Niño, S. (2017). La actividad de los participantes como fuente de información para promover la colaboración. Una analítica del aprendizaje basada en el modelo de Influencia Educativa Distribuida. $R E D$, Revista de Educación a Distancia, 53. doi: https://doi.org/10.6018/red/53/2

Cooper, H. M. (1988). Organizing knowledge syntheses: A taxonomy of literature reviews. Knowledge, Technology y Policy, 1(1), 104-126. doi: https://doi. org/10.1007/BFo3177550

Dawson, S., Gaševi, D., Siemens, G., y Joksimovic, S. (2014). Current state and future trends: A citation network analysis of the learning analytics field. En Proceedings of the fourth international conference on learning analytics and knowledge (pp. 231-240). ACM. doi: https://doi. org/10.1145/2567574.2567585

Dillenbourg, P., Järvelä, S., y Fischer, F. (2009). The evolution of research on computer-supported collaborative learning: from design to orchestration. En N. Balacheff, S. Ludvigsen, T. de Jong, A. Lazonder y S. Barnes (Eds.), TechnologyEnhanced Learning (pp. 3-19). Springer. doi: https://doi.org/10.1007/978-14020-9827-7 1

Dimitracopoulou, A. (2008). Computer based Interaction Analysis Supporting Selfregulation: Achievements and Prospects of an Emerging Research Direction. Technology, Instruction, Cognition and Learning, 6(4), 291-314. Recuperado de http://www.oldcitypublishing.com FullText/TICLfulltext/TICL6.4fulltext/ TICLv6n4p291-314Dimitracopoulou.pdf

Engel, A. (2008). Construcción del conocimiento en entornos virtuales de enseñanzay aprendizaje. La interrelación entre los procesos de colaboración entre alumnos y los procesos de ayuda y guía del profesor. (Tesis doctoral, Universidad de Barcelona). Recuperado de http:/ www.tesisenxarxa.net/TDX-0123109115623

Ferguson, R. (2012). Learning analytics: drivers, developments and challenges. International Journal of Technology Enhanced Learning, 4(5/6), 304. doi: https://doi.org/10.1504 IJTEL.2012.051816

Fessakis, G., Dimitracopoulou, A., y Palaiodimos, A. (2013). Graphical interaction analysis impact on groups collaborating through blogs. Educational Technology and Society, 16(1), 24.3-253. Recuperado de http://www.ifets.info/ journals/16 1/21.pdf

García, E., Romero, C., Ventura, S., y de Castro, C. (2011). A collaborative educational association rule mining tool. The Internet and Higher Education, 14(2), 77-88. doi: http://doi.org/10.1016/j. iheduc.2010.07.006

Janssen, J., y Bodemer, D. (2013). Coordinated Computer-Supported Collaborative Learning: Awareness and Awareness Tools. Educational Psycholoqist, 48(1), 40-55. doi: https:/ doi.org/10.1080/00461520.2012.749153 Järvelä, S., Kirschner, P. A., Panadero, E., Malmberg, J., Phielix, C., Jaspers, J., y Järvenoja, H. (2015). Enhancing socially shared regulation in collaborative learning groups: designing for CSCL regulation tools. Educational Technology Research and Development, 63, 125-142. 
doi: https://doi.org/10.1007/s11423-0142358-1

Jeong, H., y Hmelo-Silver, C. E. (2016). Seven affordances of computersupported collaborative learning: How to support collaborative learning? How can technologies help? Educational Psychologist, 51(2), 247-265. doi: https:/ doi.org/10.1080/00461520.2016.115865 4

Jeong, H., Hmelo-Silver, C. E., y Yu, Y. (2014). An examination of CSCL methodological practices and the influence of theoretical frameworks 2005-2009. International Journal of Computer-Supported Collaborative Learning, 9(3), 305-334. doi: https://doi.org/10.1007/s11412-0142198-3

Kimmerle, J., y Cress, U. (2008). Group awareness and self-presentation in computer-supported information exchange. International Journal of Computer-Supported Collaborative Learning, 3(1), 85-97. doi: https://doi. org/10.1007/s11412-007-9027-Z

Kirschner, P. A., y Erkens, G. (2013). Toward a Framework for CSCL Research. Educational Psychologist, 48(1), 1-8. doi: https://doi.org/10.1080/00461520.2012 750227

Kuosa, K., Distante, D., Tervakari, A., Cerulo, L., Fernández, A., Koro, J., y Kailanto, M. (2016). Interactive visualization tools to improve learning and teaching in online learning environments. International Journal of Distance Education Technologies, 14(1), 1-21. doi: https://doi. org/10.4018/IJDET.2016010101

Kwon, K., Hong, R.-Y., y Laffey, J. M. (2013). The educational impact of metacognitive group coordination in computersupported collaborative learning. Computers in Human Behavior, 29(4), 1271-1281. doi: https://doi.org/10.1016/i. chb.2013.01.003

Marzouk, Z., Rakovic, M., Liaqat, A., Vytasek, J., Samadi, D., Stewart-Alonso, J., ... y
Nesbit, J. C. (2016). What if learning analytics were based on learning science? Australasian Journal of Educational Technology, 32(6). doi: https://doi. org/10.14742/ajet.3058

McCormick,J.(2013).Visualizinginteraction: Pilot investigation of a discourse analytics tool for online discussion. Bulletin of the Technical Committee on Learning Technology, 15(2), 10-13. Recuperado de http://lttf.ieee.org/issues/april2013/ McCormick.pdf

Mora, N., Caballé, S., y Daradoumis, T. (2016). Providing a multi-fold assessment framework to virtualized collaborative learning in support for engineering education. International Journal of Emerging Technologies in Learning, 11(7). doi: https://doi.org/10.3991/ijet. v11io7.5882

Niño, S. (2017). El uso de la información sobre elejercicio de la influencia educativa para la mejora de los procesos y los resultados del aprendizaje colaborativo en entornos digitales. (Tesis doctoral, Universidad de Barcelona). Recuperado de http://psyed.edu.es/archivos/grintie/ Tesis SNino junio17.pdt

Park, Y., y Jo, I.-H. (2015). Development of the learning analytics dashboard to support students' learning performance. Journal of Universal Computer Science, 21(1), 110-133. doi: https://doi. org/10.3217/jucs-021-01-0110

Pifarré, M., Cobos, R., y Argelagós, E. (2014). Incidence of group awareness information on students' collaborative learning processes. Journal of Computer Assisted Learning, 30(4), 300-317. doi: https://doi.org/10.1111/jcal.12043

Resta, P., y Laferrière, T. (2007). Technology in support of collaborative learning. Educational Psychology Review, 19(1), 65-83. doi: https://doi.org/10.1007/ s10648-007-9042-7

Romero, C., y Ventura, S. (2010). Educational data mining: A review of the state of the 
art. IEEE Transactions on Systems, Man and Cybernetics Part C: Applications and Reviews, 40, 601-618. doi: https://doi. org/10.1109/TSMCC.2010.2053532

Roschelle, J. (2013). Special Issue on CSCL: Discussion. Educational Psycholoqist, 48, 67-70. doi: https://doi.org/10.1080/004 61520.2012 .749445

Sangin, M., Molinari, G., Nüssli, M.A. M.-A., y Dillenbourg, P. (2011). Facilitating peer knowledge modeling: Effects of a knowledge awareness tool on collaborative learning outcomes and processes. Computers in Human Behavior, 27(3), 1059-1067. doi: https:// doi.org/10.1016/j.chb.2010.05.032

Schoor, C., Kownatzki, S., Narciss, S., y Körndle, H. (2014). Effects of feeding back the motivation of acollaboratively learning group. Electronic Journal of Research in Educational Psycholoqu, 12(1), 191210. doi: https://doi.org/10.14204/ ejrep.32.13077

Siemens, G., y Long, P. (2011). Penetrating the Fog: Analytics in Learning and Education. EDUCAUSE Review, 46, 30-32. https:// doi.org/10.1145/2330601.2330605

Silius, K., Tervakari, A.-M. A.-M., y Kailanto, M. (2013). Visualizations of User Data in a Social Media Enhanced Web-based Environment in Higher Education. International Journal of Emerging Technologies in Learning, 8(S2), 13. doi: https://doi.org/10.3991/ijet.v8iS2.2740

Stahl, G. (2015). A decade of CSCL. International Journal of Computer-
Supported Collaborative Learning, 10(4), 337-344. doi: https://doi.org/10.1007/ s11412-015-9222-2

Stahl, G., Koschmann,T.,ySuthers, D. (2006). Computer-supported collaborative learning: An historical perspective. En R. K. Sawyer (Ed.), Cambridge handbook of the learning sciences (pp. 409-426). Cambridge, UK: Cambridge University Press. Recuperado de http://GerryStahl. net/cscl/CSCL English.pdf

Wise, A. F., Vytasek, J., Hausknecht, S., y Zhao, Y. (2016). Developing learning analytics design knowledge in the "middle space": The student tuning model and align design framework for learning analytics use. Journal of Asynchronous Learning Network, 2O(2). Recuperado de https://olj.onlinelearningconsortium. org/index.php/olj/article/view/783/210

Xia, L. (2015). Reflections on on-line teaching and learning, based on learning process data. World Transactions on Engineering and Technology Education, 13(3). Recuperado de http://www.wiete. com.au/journals/WTE\&TE/Pages/ Vol.13,\%20No.3\%20(2015)/40-Xia-L.pdf

Zorrilla, M., Álvarez, E., y García-Saiz, D. (2015). A parametrisable method for measuring online attendance in e-learning tools. International Journal of Technology Enhanced Learning, 7(4), 289-308. doi: https://doi.org/10.1504/ IJTEL.2015.074185

\section{PERFIL ACADÉMICO Y PROFESIONAL DEL AUTOR/ES}

César Coll Salvador. Doctor en Psicología y catedrático de Psicología de la Educación del Departamento de Cognición, Desarrollo y Psicología de la Educación de la Universidad de Barcelona, España. Investigador principal del Grupo de Investigación en Interacción e Influencia Educativa (GRINTIE). Coordinador general del programa de Doctorado Interuniversitario de Psicología de la Educación. E-mail:ccoll@ub.edu 
Anna Engel Rocamora. Doctora en Psicología y profesora agregada del Departamento de Cognición, Desarrollo y Psicología de la Educación en la Universidad de Barcelona, España. Miembro del grupo de investigación GRINTIE. E-mail: anna.engel@ub.edu

\section{DIRECCIÓN DE LOS AUTORES}

Facultad de Psicología

Universidad de Barcelona

Edificio de Ponent

Vall d'Hebron, 171

o8035 Barcelona, España

Shamaly Niño Carrasco. Doctora en Psicología de la Educación por la Universidad de Barcelona, España. Profesora de la Facultad de Ciencias Humanas en la Universidad Autónoma de Baja California, México. Colaboradora del grupo de investigación GRINTIE.

E-mail: shamaly.nino@gmail.com

\section{DIRECCIÓN DE LA AUTORA}

Facultad de Ciencias Humanas

Universidad Autónoma de Baja California

Bulevar Castellón y Lombardo Toledano s/n

Conjunto Urbano Esperanza

21350 Mexicali, Baja California, México

Fecha de recepción del artículo: 12/12/2017

Fecha de aceptación del artículo: 17/01/2018

\section{Como citar este artículo:}

Coll Salvador, C., Engel Rocamora, A., Niño Carrasco, S. (2018). Uso de los registros de actividad para mejorar la colaboración en línea. RIED. Revista Iberoamericana de Educación a Distancia, 21(2), pp. 139-157. doi: http://dx.doi. org/10.5944/ried.21.2.20641 